\title{
Multi-directional plasmonic splitter and polarization analyzer based on the catenary metasurface
}

\section{Panpan Chen ( $\sim 2691854592 @ q q . c o m$ )}

Anhui Normal University School of Physics and Electronic Information https://orcid.org/0000-00021385-7949

\section{Cong Chen}

Anhui Normal University School of Physics and Electronic Information

\section{Jianxin Xi}

Anhui Normal University School of Physics and Electronic Information

\section{Xiang Du}

Anhui Normal University School of Physics and Electronic Information

\section{Li Liang}

Anhui Normal University School of Physics and Electronic Information

\section{Jiajia Mi}

Anhui Normal University School of Physics and Electronic Information

Jianping Shi

Anhui Normal University School of Physics and Electronic Information

\section{Research Article}

Keywords: Surface plasmon polaritons, Beam splitters, Catenary, Metasurface

Posted Date: May 27th, 2021

DOl: https://doi.org/10.21203/rs.3.rs-358767/v1

License: (c) (1) This work is licensed under a Creative Commons Attribution 4.0 International License. Read Full License

Version of Record: A version of this preprint was published at Plasmonics on July 14th, 2021. See the published version at https://doi.org/10.1007/s11468-021-01482-x. 


\title{
Multi-directional plasmonic splitter and polarization analyzer based on the catenary metasurface
}

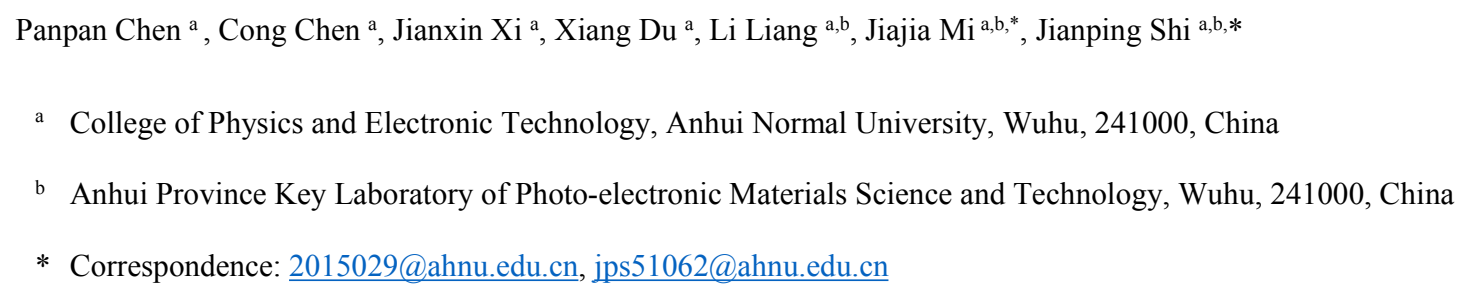

\begin{abstract}
Owing to the unique properties of strongly confined and enhanced electric fields, surface plasmon polaritons (SPPs) provide a new platform for the realization of ultracompact plasmonic circuits. However, there are challenges in coupling light into SPPs efficiently and subsequently routing SPPs. Here, we propose a multi-directional SPPs splitter and polarization analyzer based on the catenary metasurface. Based on the abundant electromagnetic modes and geometric phase modulation principle of catenary structure, the device has realized high efficiency beam splitting for four different polarization states ( $x$-polarization, $y$-polarization, LCP and RCP). The central wavelength of the device is $632 \mathrm{~nm}$ and the operation bandwidth can reach $70 \mathrm{~nm}$ (585-655 nm). Based on the phenomenon of SPPs beam splitting, we present a prototype of a polarization analyzer, which can detect the polarization state of incident light by adding photodetector with light intensity logic threshold in four directions. Moreover, by combining this device with dynamic polarization modulation techniques, it is possible to be served as a router or switch in integrated photonic circuits.
\end{abstract}

Keywords: Surface plasmon polaritons; Beam splitters; Catenary; Metasurface 


\section{Introduction}

Surface plasmon polaritons (SPPs) [1, 2] with remarkable capabilities of subwavelength field confinement and strong field enhancement have provided an efficient route to realize on-chip optical devices such as SPPs splitter, directional coupler and ultrasensitive sensor [3-11]. Among them, the SPPs splitter, which can separate the incident beams to propagate in different directions, plays an important role in the router and Mach-Zehnder interferometers (MZIs) [12-14].

Recently, due to the requirement of ultra-diffraction limit spectroscopy, a variety of SPPs splitters have been designed by using asymmetric double grooves, T-shaped, and composite slits and grooves [15-17]. However, the aforementioned SPPs splitter can only excite SPPs in different directions under different wavelengths of the same polarized light. In order to excite SPPs with different directions under different polarized lights, Lin et al. proposed multiple parallel column nanostructure pairs [18], which realized the SPPs beam splitting under the incidence of left-handed circularly polarized (LCP) and right-handed circularly polarized (RCP). Sun et al. designed a hybrid strip waveguide, the two incident waveguide modes of orthogonal polarization states can be efficiently split on chips based on the different radiation losses [19].

However, we note here that the polarization-controlled SPPs excitation examined thus far depend either on $x$-polarized and $y$-polarized incidences or on LCP and RCP incidences, only two polarization states of incident light can be splitted in one structure [20-22]. In order to address this problem, Shen et al. demonstrated a bi/tridirectional SPPs splitter based on a slanted gold mushroom array [23], achieving different responses to multiple incident light polarization states. $\mathrm{Xu}$ et al. proposed a metasurface composed of two split-ring-shaped slit resonators, which realized different asymmetric excitation under the $x$-polarized, $y$-polarized, LCP, and RCP incidences [24]. However, the unit structures of the above devices are not continuous, so there are abundant structural parameters to be considered, and the processing technology is complicated, which relatively limits their practical applications. More importantly, it is quite a challenge to achieve different responses of multiple 
polarization states with single unit structure since linearly (circularly) polarized light can be regarded as a superposition of two circularly (linearly) polarized lights [25].

The catenary curve whose shape is similar to the free-hanging chain with uniform force at each point has been widely used in the micro-nano optics [26-30]. Owing to the abundant electromagnetic modes and geometric phase modulation ability of the catenary apertures, the SPPs directional launching can be modulated under the incidence of both linearly and circularly polarized lights with different directional excitation mechanisms [31-33]. Therefore, complex SPPs response can be achieved by using the unit catenary aperture, then considering the remarkable ability of metasurface in light manipulation [34-37], the SPPs with different directions under different incident light polarization states can be realized by designing a reasonable metasurface. In this paper, we propose a multi-directional SPPs splitter and polarization analyzer based on the catenary metasurface, which can modulate SPPs directions by changing the incident polarization state. So this device has the performance of multidirectional splitter and polarization analyzer simultaneously. The results show the potential of the catenary metasurface in subwavelength optical devices and integrated optical circuits.

\section{Device structure and design}

To gain insight into the optical properties of the multi-directional SPPs splitter based on the catenary metasurface, we performed numerical simulations using the finite-difference time-domain (FDTD) method. Here, the perfectly matched layer (PML) boundary condition is adopted to eliminate unwanted deviation. The schematic of the proposed multi-directional SPPs splitter based on the catenary metasurface is depicted in Figure 1a. Here, the catenary aperture is obtained by translating the catenary curve with $\Delta$ along the $X$ direction and the catenary curve is expressed as:

$$
x=\frac{\Lambda}{\pi} \ln \left|\sec \left(\frac{\pi y}{\Lambda}\right)\right|
$$

where $\Lambda$ is the width of the catenary in the $Y$ direction. Because the value of equation (1) is infinite for $y= \pm \Lambda / 2$, catenary curves are truncated at the two 
ends in practical design. In this paper, the intersection of two catenary curves is set at $y= \pm 0.46 \Lambda$, therefore the width of catenary apertures along the $Y$ direction is $0.92 \Lambda$. The optimized parameters are $0.92 \Lambda=552 \mathrm{~nm}, \Delta=130 \mathrm{~nm}$. And the thickness of the gold film is $120 \mathrm{~nm}$, the relative permittivity of gold as a function of the wavelength is taken from the data of Palik [38].

To enhance the beam splitting and overall SPPs field at the designed wavelength of $632 \mathrm{~nm}$, a $4 \times 4$ array setup is adopted. Here, the periods in the $X$ and $Y$ directions are Tx and Ty, respectively. And the periods should set to be $600 \mathrm{~nm}$ along both the $X$ and $Y$ directions so that the excited SPPs on the air side could constructively interfere with each other [39]. The whole structure is normally illuminated from the back side using the $x$-polarized, $y$-polarized, LCP, and RCP light, respectively.
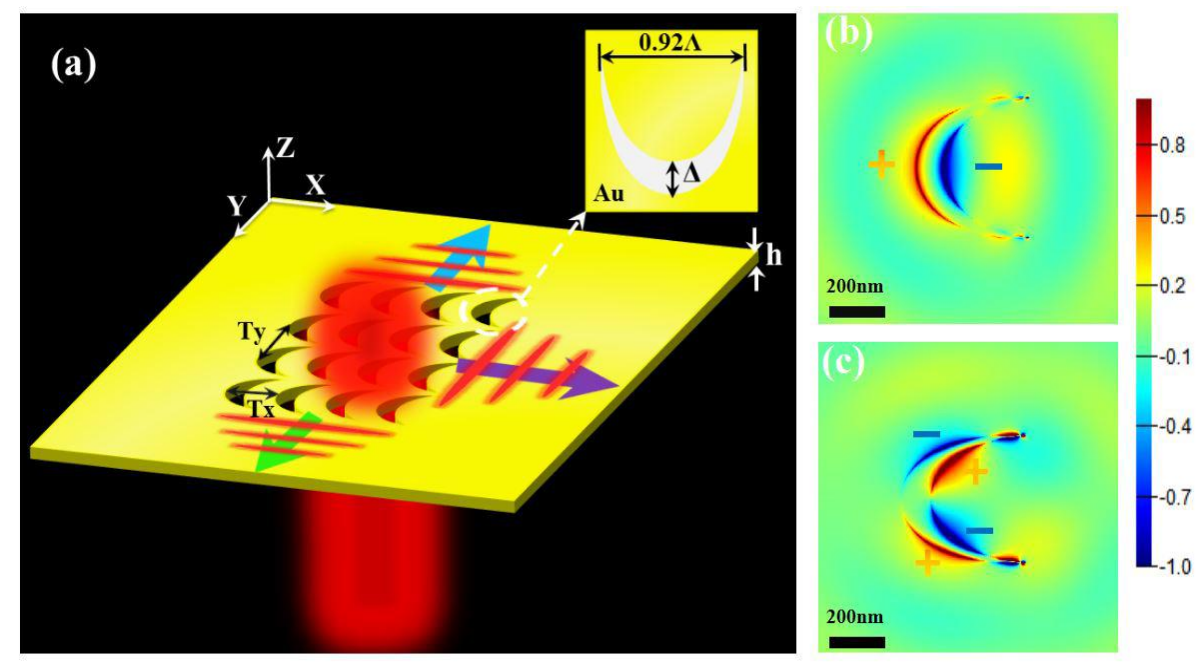

Figure 1. (a) The schematic of the multi-directional SPPs splitter based on the catenary metasurface. The inset represents the cocrresponding unit catenary aperture. The electric field patterns in the catenary aperture under different polarized incidence at the wavelength of 632 nm (b) $x$-polarized and (c) $y$-polarized. Inset: “+” and "-" indicate the charge distributions.

To the best of our knowledge, researchers have realized directional excitation of SPPs under LCP and RCP based on the geometric phase modulation theory of the catenary $[31,32]$. Besides, through the analysis of the exciting electromagnetic mode in catenary apertures, our research group realized the SPPs directional excitation under linearly polarized light on the basis of mode superposition theory [33]. Therefore, through the combination of the exciting electromagnetic mode of the 
catenary aperture and geometric phase modulation theory, it is of great potential in controlling the direction of the excited SPPs under the illumination of both linearly and circularly polarized light, which provides the feasibility for the realization of SPPs splitter.

When the linearly polarized light is illuminated on the catenary metasurface, there are abundant and different electromagnetic modes in a single catenary aperture, as seen in Figures $1 \mathrm{~b}$ and 1c. The electric field distribution indicates a dipole mode and a quadrupole mode under the $x$-polarized and $y$-polarized incidence at the wavelength of $632 \mathrm{~nm}$, respectively. Because of the asymmetry of the catenary aperture in the $X$ direction, the electric field intensity on the left and right side of the catenary aperture is different and the electric field on the right side is stronger than that of the left side, thus the SPPs excitation in the $+X$ direction can be realized. And for the $y$-polarized incidence, owing to the symmetry of the catenary aperture in the $Y$ direction, the excited SPPs are symmetrically distributed in the $\pm Y$ direction. Therefore, the abundant electromagnetic modes have contributed to achieving efficient SPPs directional excitation.

For circularly polarized light, owing to the spin-orbit interactions of light $[40,41]$, the geometric phase of the excited SPP is $2 \sigma \zeta(y)$, where $\sigma= \pm 1$ denotes LCP and RCP. According to the equation of catenary curve, the inclination angle can be expressed as follows: $\zeta(\mathrm{y})=\pi y / \Lambda$. Take the incident LCP as an example, in order to make the excited SPPs constructively interfere along the $+Y$ direction, the superimposing of the geometric phase and propagation phase retardation of the excited SPPs need to satisfy the phase condition: $-k_{s p p} y_{0}+2 \zeta=0$, where $k_{s p p}$ is the wave vector of the SPPs, and $y_{0}$ is the position of the metasurface. Therefore, given the relationship between the inclination angle of the catenary curve and the geometric phase shift of the excited SPPs, the catenary aperture with accurate designed parameters can be applied to modulate the directions of SPPs propagation under the normal illumination of circularly polarized light.

Because the SPPs excited by different polarized lights have specific propagation 
directions and the size of the catenary aperture is smaller than the incident wavelength, it can be regarded as a directional point source. According to Huygens-Fresnel principle [42], through the coherent superposition of arrays of directional point sources, the plane wave propagating in different directions is obtained. Based on the above principles, a SPPs splitter by using the catenary metasurface can be realized.

\section{Simulation results and analysis}

\subsection{Multi-directional SPPs splitter}

To demonstrate the design of the SPPs splitter, the electric field amplitude $|\mathrm{Ez}|$ on the top surface of the catenary metasurface is calculated for the $x$-polarized, $y$-polarized, LCP, and RCP excitation at the wavelength of $632 \mathrm{~nm}$, and the corresponding results are shown in Figure 2, respectively. The Ez-feld is depicted since this field component is dominantly related to the in-plane propagation of the SPPs [43].
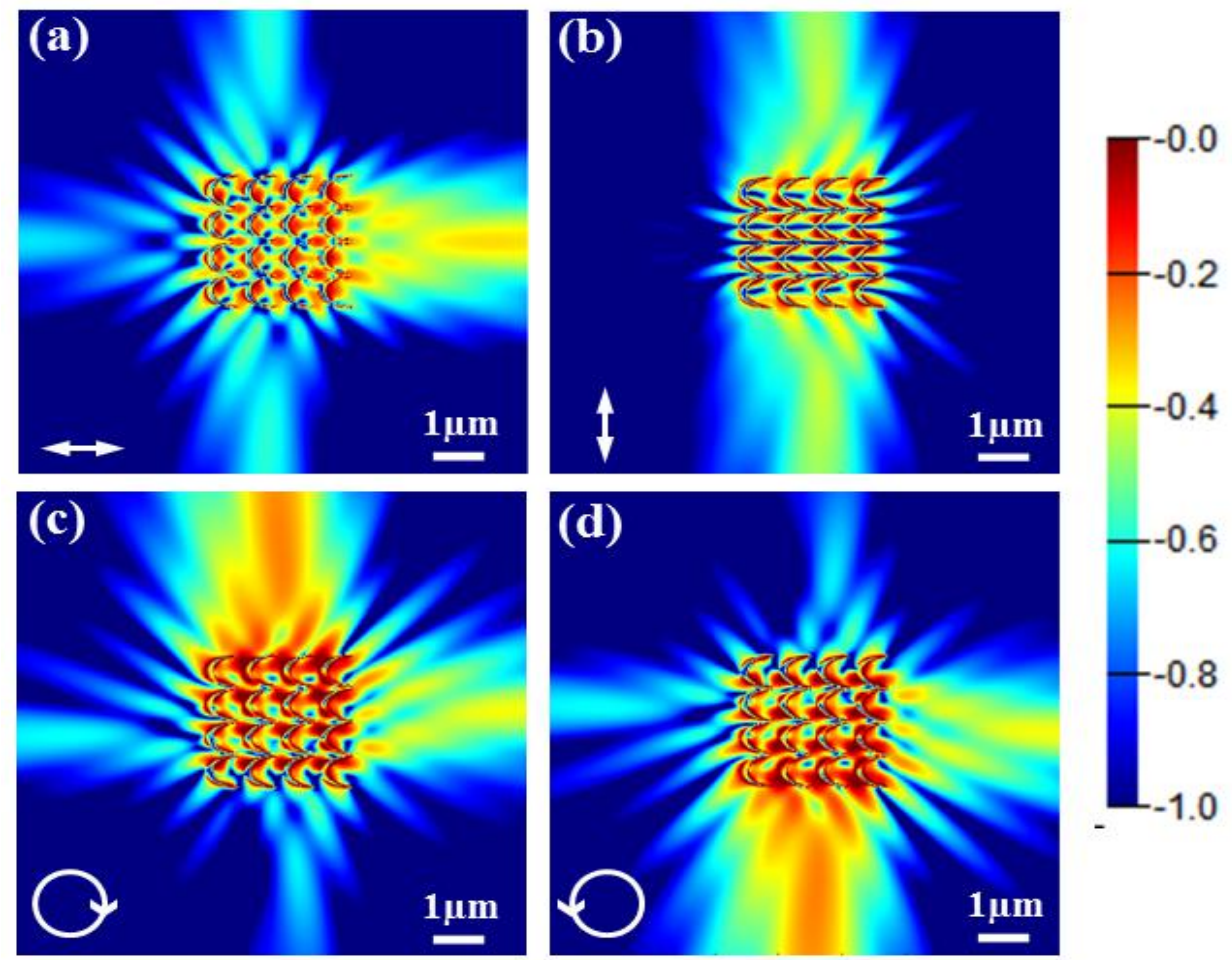

Figure 2. Simulated Ez-field-amplitude distributions at $632 \mathrm{~nm}$ of the catenary metasurface under the $x$-polarized(a), $y$-polarized(b), LCP(c), and $\mathrm{RCP}(\mathrm{d})$ incidences, respectively. The inset arrow in the bottom-left corner of each picture represents the corresponding incident polarization state. 
When the $x$-polarized light is illuminated on the catenary metasurface, the excited SPPs electric field mainly propagate along the $+X$ direction, whereas there are few SPPs along other directions as shown in Figure 2a. When the $y$-polarized light is illuminated, the SPPs are only excited along the $Y$ direction, the intensity of the SPPs is close to 0 in the $X$ direction (Figure 2b). And for two circularly polarized incident lights, the catenary metasurface couples the LCP light into the SPPs propagating along the $+X$ and $+Y$ directions, and the SPPs can hardly be excited along the $-X$ and $-Y$ direction (Figure 2c). While for the RCP incident light, the coupled SPPs propagate along the $+X$ and $-Y$ directions (Figure 2d). It can be seen that the launched SPPs are obviously different from each other depending on the polarization state of the incident light, as can be gathered from the presented field distributions. Therefore, the multi-directional SPPs splitter based on the catenary metasurface is confirmed.

In order to quantitatively describe the the SPPs beam splitting effect, we evaluated the coupling efficiency in the wavelength range from 550 to $750 \mathrm{~nm}$ by integrating the field strength along metal/air interface. Here, the coupling efficiency is defined as the ratio of the field strength launched in different directions to the incoming field strength. The corresponding coupling efficiency along different directions based on the catenary metasurface under the $x$-polarized, $y$-polarized, LCP, and RCP incidences are shown in Figure 3, respectively.

Figure 3 a shows the coupling efficiency along different directions under the illumination of $x$-polarized light. It can be seen that the coupling efficiency in the $+X$ direction is greater than that in other three directions. And the coupling efficiency in the $+X$ direction is higher than $10 \%$ in the wavelength range of $570-710 \mathrm{~nm}$, while it is lower than $10 \%$ in other directions. And for $y$-polarized light, as shown in Figure $3 b$, the coupling efficiency along the $Y$ direction is more than $10 \%$ in the wavelength range of $585-655 \mathrm{~nm}$, while the coupling efficiency in the $X$ direction is close to zero.

When the catenary metasurface is illuminated by LCP (RCP), as shown in 
Figure $3 \mathrm{c}(3 \mathrm{~d})$, the coupling efficiency propagating along the $+X$ and $+Y(-Y)$ directions is more than $10 \%$ from $570-685 \mathrm{~nm}$, while the coupling efficiency of SPPs propagating in the $-X$ and $-Y(+Y)$ directions is low. Moveover, the coupling efficiency is less than 5\% near the wavelength of $632 \mathrm{~nm}$, which obviously suppresses SPPs excitation.
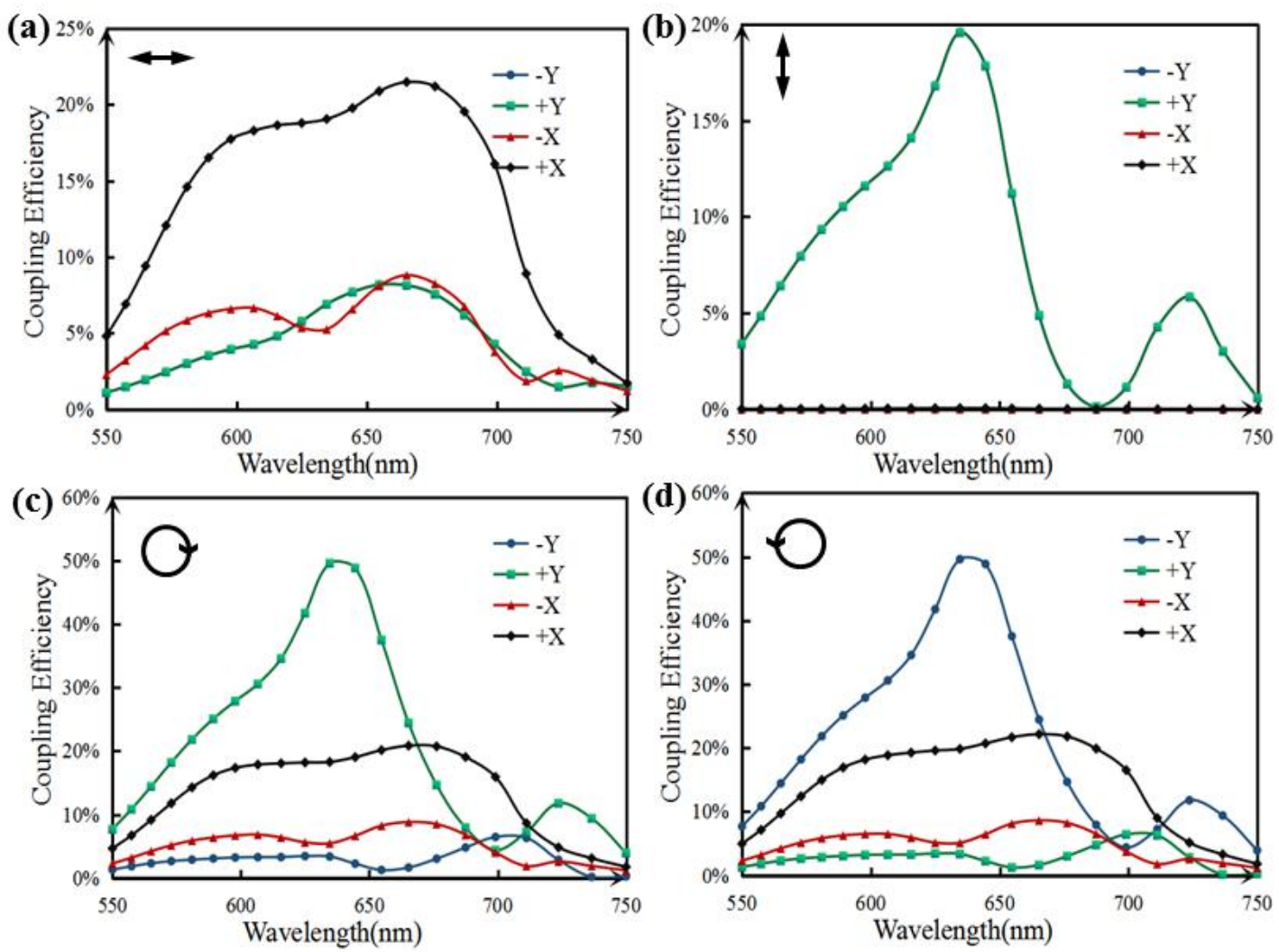

Figure 3. Simulated coupling efficiency in the wavelength range from 550 to $750 \mathrm{~nm}$ of the catenary mrtasurface under the $x$-polarized(a), $y$-polarized(b), LCP(c), and RCP(d) incidences, respectively.

The coupling efficiency in Figure 3 clearly shows the different coupling efficiency in different directions based on the catenary metasurface under different polarized light incidences. In particular, the coupling efficiency of the four polarization states all have peaks in one or two directions near the wavelength of $632 \mathrm{~nm}$, while the excitation of SPPs is suppressed in other directions. Therefore, the results show that the SPPs beam splitting can be achieved by using the catenary metasurface.

\subsection{Prototype of on-chip polarization analyzer}

Based on the above beam splitting phenomenon, a prototype of an on-chip 
polarization detector is designed, as shown in Figure 4a. Here, the catenary metasurface is in the middle of the device and four photodetectors are placed in four directions ( $+X$ direction, $-X$ direction, $-Y$ direction and $+Y$ direction), which are used to collect the intensity of light. For linear interference scheme, the logic states " 1 " and " 0 " in the output ports of the optical logic gate are usually defined by the intensity of the output optical signals [44]. Here, according to the coupling efficiency of the excited SPPs under different polarized light incidences, we define the logical state " 1 " of the photodetectors as the coupling efficiency greater than $10 \%$, and the logical state " 0 " as the coupling efficiency less than $10 \%$.

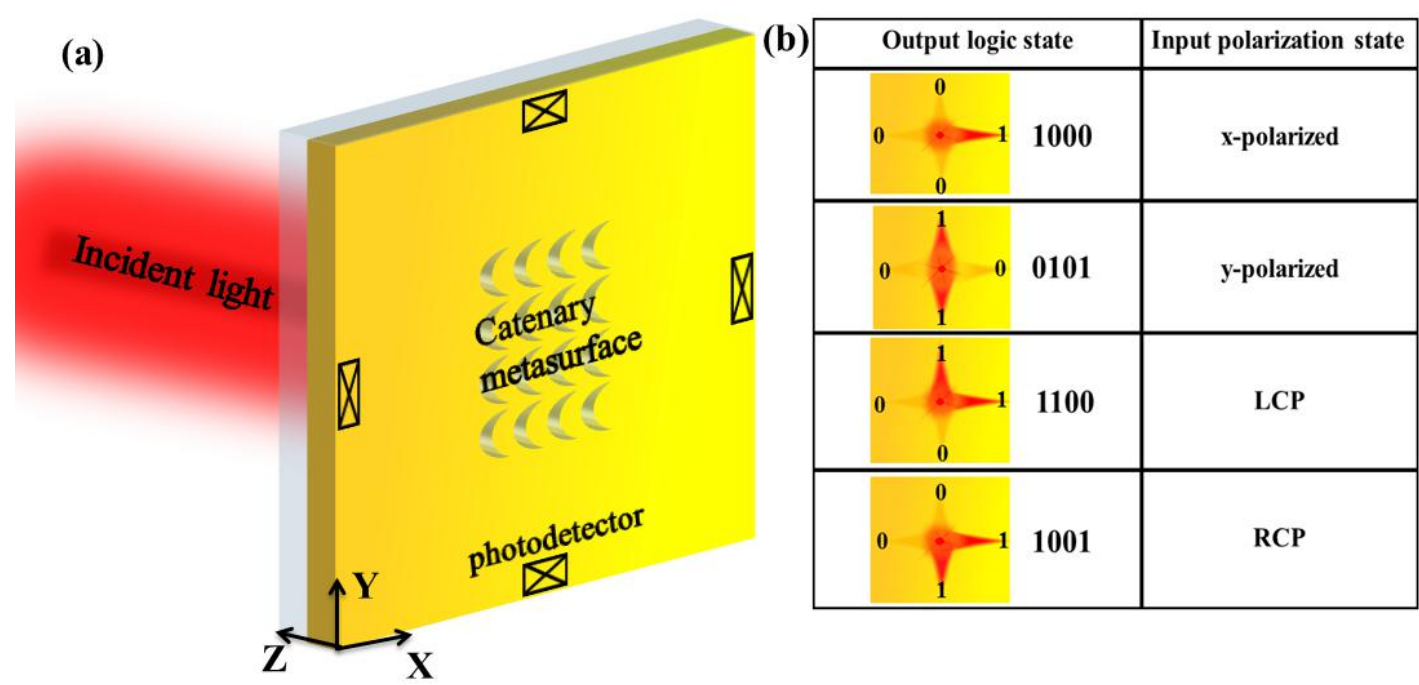

Figure 4. (a) The schematic of on-chip polarization detector prototype. The black rectangles represent the photodetectors and (b) results of the logic gates.

To achieve logic operation, the output of the logic gate is required to vary with the input polarization state [45]. Therefore, combining with the SPPs coupling efficiency and the $|\mathrm{Ez}|$ electric field distribution, we summarize the results of the logic gates in Figure 4b. When the output logic state is "1000", it means that the incident light is $x$-polarized and when the output logic state is "0101", it indicates that the incident light is $y$-polarized. Similarly, the corresponding logic state of RCP is "1001" and the output logic state of LCP is "1100". Therefore when the light is normally illuminated on the catenary metasurface, we can quickly determine the polarization state of the input light 
according to the logic state of the output. Besides, the results show that the device is valid in a bandwidth as large as $70 \mathrm{~nm}(585-655 \mathrm{~nm})$, which brings great advantages to the proposed polarization analyzer in practical applications.

\section{Conclusion}

In summary, we have designed and simulated the multi-directional SPPs splitter and polarization analyzer based on the catenary metasurface. By employing abundant electromagnetic modes and geometric phase modulation ability of the catenary metasurface, SPPs can be excited and propagate in different directions under the illumination of different polarized lights. Moreover, the device can be regarded as a polarization analyzer corresponding to the coupling efficiency of the SPPs launched in each direction. Furthermore, the device is applicable to a broad bandwidth of $70 \mathrm{~nm}$. Therefore, the proposed catenary metasurface points out a new way to achieve ultra-compact optical circuits.

Funding: This work was supported by the National Natural Science Foundation of China (Grant Nos. 61775002).

Conflicts of Interest: The authors declare no conflicts of interest.

Availability of data and material: The datasets generated during and/or analyzed during the current study are available from the corresponding author on reasonable request.

Code availability : Not applicable

Authors' contributions : All authors contributed to the study conception and design. Material preparation, data collection and analysis were performed by Panpan Chen, Cong Chen, Jianxin Xi, Xiang Du, Li Liang, Jiajia M and Jianping Shi. The first draft of the manuscript was written by Panpan Chen and all authors commented on previous versions of the manuscript. All authors read and approved the final manuscript. 


\section{References}

[1] Barnes WL, Dereux A, Ebbesen TW (2003) Surface plasmon subwavelength optics. Nature 424:824-830. https://doi.org/10.1038/nature01937.

[2] Maier SA (2007) Plasmonics: Fundamentals and Applications. Springer-Verlag.

[3] Gramotnev DK, Bozhevolnyi SI (2010) Plasmonics beyond the diffraction limit. Nat. Photonics 4:83-91. https://doi.org/10.1038/nphoton.2009.282.

[4] Wang L, Hasanzadeh M, Meunier M (2020)Optical Properties and Applications of Plasmonic-Metal Nanoparticles. Adv. Funct. Mater. 2005400. https://doi.org/10.1080/15421400590946415.

[5] Gan Q, Guo B, Song G, Chen L, Fu Z, Ding YJ, Bartoli FJ (2007) Plasmonic surface-wave splitter. Appl. Phys. Lett. 90:161130. https://doi.org/10.1063/1.2731524. [6] He P, Zhang H, Gao X, Niu L, Tang W, Lu J, Zhang L, Cui T (2019) A novel spoof surface plasmon polariton structure to reach ultra-strong field confinements. Opto-Electron. Adv. 2 :190001. DOI:10.29026/oea.2019.190001.

[7] Guo Y, Zhang Z, Pu M, Huang Y, Li X, Ma X, Xu M, Luo X (2019) Spoof plasmonic metasurfaces with catenary dispersion for two-dimensional wide-angle focusing and imaging. Iscience 21: 145-156. https://doi.org/10.1016/j.isci.2019.10.019.

[8] Huang L, Chen X, Bai B, Tan Q, Jin G, Zentgraf T, Zhang S (2013) Helicity dependent directional surface plasmon polariton excitation using a metasurface with interfacial phase discontinuity. Light: Sci. Appl. 2:e70. https://doi.org/10.1038/lsa.2013.26.

[9] Lee SY, Yun H, Lee Y, Lee B (2014) Switchable surface plasmon dichroic splitter modulated by optical polarization. Laser \& Photonics Reviews 8:777-784. https://doi.org/10.1002/lpor.201400025.

[10] Sun W, He Q, Sun S, Zhou L (2016) High-efficiency surface plasmon meta-couplers: concept and microwave-regime realizations. Light: Sci. Appl. 5: e16003. https://doi.org/10.1038/1sa.2016.3.

[11] Vazquez-Mena O, Sannomiya T, Villanueva LG, Voros J, Brugger J (2011) 
Metallic nanodots by stencil lithography for plasmonic biosensing applications. Acs Nano 5:844-853. https://doi.org/10.1021/nn1019253.

[12] Augustin LM, Hanfoug R, van der Tol JJGM, de Laat WJM, Smit MK (2007) A Compact Integrated Polarization Splitter/Converter in InGaAsP-InP. IEEE Photonics Technology Letters 19:1286-1288. doi: 10.1109/LPT.2007.902277.

[13] Wahsheh RA, Lu Z, Abushagur MAG (2009) Nanoplasmonic couplers and splitters. Opt. Express 17:19033-19040. https://doi.org/10.1364/OE.17.019033.

[14] Yuan G, Wang P, Lu Y, Ming H (2009) Multimode interference splitter based on dielectric-loaded surface plasmon polariton waveguides. Opt. Express 17: 12594-12600. https://doi.org/10.1364/OE.17.012594.

[15] Liu JSQ, Pala RA, Afshinmanesh F, Cai W, Brongersma ML (2011) A submicron $\begin{array}{llll}\text { plasmonic dichroic } & \text { splitter. }\end{array}$ https://doi.org/10.1038/ncomms1537.

[16] Zhang X, Li Z, Chen J, Yue S, Gong Q (2013) A dichroic surface-plasmon-polariton splitter based on an asymmetric T-shape nanoslit. Opt. Express 21:14548-14554. https://doi.org/10.1364/OE.21.014548.

[17] Zhang X, Li Z, Chen J, Liao H, Yue S, Gong Q (2013) A submicron surface-plasmon-polariton dichroic splitter based on a composite cavity structure. Appl. Phys. Lett. 102:091110. https://doi.org/10.1063/1.4794803.

[18] Lin J, Mueller JB, Wang Q, Yuan G, Antoniou N, Yuan XC, Capasso F (2013) Polarization-Controlled Tunable Directional Coupling of Surface Plasmon Polaritons. Science 340:331-334. https://doi.org/10.1126/science.1233746.

[19] Sun C, Rong K, Gan F, Chu S, Gong Q, Chen J (2017) An on-chip polarization splitter based on the radiation loss in the bending hybrid plasmonic waveguide structure. Appl. Phys. Lett. 111:101105. https://doi.org/10.1063/1.4997234.

[20] Bai B , Liu L, Zhou Z (2017) Ultracompact, high extinction ratio polarization beam splitter-rotator based on hybrid plasmonic-dielectric directional coupling. Opt. Lett. 42:4752-4755. https://doi.org/10.1364/OL.42.004752.

[21] Gan F, Sun C, Li H, Gong Q, Chen J (2018) On-chip polarization splitter based on a multimode plasmonic waveguide. Photonics Research 6:47-53. 
https://doi.org/10.1364/PRJ.6.000047.

[22] Zhang J, Dai J, Yang J, Hu C, Gao B, Xu Y, Zhang J (2019) Invertible plasmonic spin-hall effect at nano-scale based on u-shaped optical slot nanoantenna. Nanotechnology 30:345201-345208. DOI: 10.1088/1361-6528/ab1e8a.

[23] Shen Y, Fang G, Cerjan A, Chi Z, Fan S, Jin C (2016) Slanted gold mushroom array a switchable bi/tridirectional surface plasmon polariton splitter. Nanoscale 8:15505-15513. https://doi.org/10.1039/C6NR03488H.

[24] Xu Q, Zhang X, Yang Q, Tian C, Xu Y, Zhang J, Zhao H, Li Y, Quyang C, Tian Z, Gu J, Zhang X, Han J, Zhang W (2017) Polarization-controlled asymmetric excitation of surface plasmons. Optica 4:1044-1051. https://doi.org/10.1364/OPTICA.4.001044. [25] Ding F, Bozhevolnyi SI (2019) A Review of Unidirectional Surface Plasmon Polariton Metacouplers. IEEE Journal of Selected Topics in Quantum Electronics 25:4600611. doi: 10.1109/JSTQE.2019.2894067.

[26] Pu M, Li X, Ma X, Wang Y, Zhao Z, Wang C, Hu C, Gao P, Huang C, Ren H, Li X, Qin F, Yang J, Gu M, Hong M, Luo X (2015) Catenary optics for achromatic generation of perfect optical angular momentum. Sci. Adv. 1: e1500396. https://doi.org/10.1126/sciadv.1500396.

[27] Li X, Pu M, Zhao Z, Ma X, Jin J, Wang Y, Gao P, Luo X (2016) Catenary nanostructures as compact Bessel beam generators. Sci. Rep. 6:20524. https://doi.org/10.1038/srep20524.

[28] Guo Y, Ma X, Pu M, Li X, Zhao Z, Luo X (2018) Polarization-Controlled Broadband Accelerating Beams Generation by Single Catenary-Shaped Metasurface. Adv. Optical Mater. 6:1800592. https://doi.org/10.1002/adom.201800592.

[29] Luo X, Pu M, Li X, Ma X (2017) Broadband spin Hall effect of light in single nanoapertures. Light: Sci. Appl. 6:e16276. https://doi.org/10.1038/1sa.2016.276.

[30] Luo X, Pu M, Guo Y, Li X, Zhang F, Ma X (2020) Catenary Functions Meet Electromagnetic Waves: Opportunities and Promises. Adv. Optical Mater. 8:2001194. https://doi.org/10.1002/adom.202001194.

[31] Guo Y, Pu M, Li X, Ma X, Luo X (2018) Ultra-broadband spin-controlled directional router based on single optical catenary integrated on silicon waveguide. 
Appl. Phys. Express 11:092202. https://doi.org/10.7567/APEX.11.092202.

[32] Jin J, Li X, Guo Y, Pu M, Gao P, Ma X, Luo X (2019) Polarization-controlled unidirectional excitation of surface plasmon polaritons utilizing catenary apertures. Nanoscale 11:3952-3957. https://doi.org/10.1039/C8NR09383K.

[33] Chen P, Chen C, Qin S, Xi J, Huang W, Shi F, Li K, Liang L, Shi J (2020) Efficient planar plasmonic directional launching of linearly polarized light in a catenary metasurface. Phys. Chem. Chem. Phys. 22:27554 - 27559. https://doi.org/10.1039/D0CP05095D.

[34] Deng L, Deng J, Guan Z, Tao J, Chen Y, Yang Y, Zhang D, Tang J, Li Z, Li Z, Yu S, Zheng G, Xu H, Qiu C, Zhang S (2020) Malus-metasurface-assisted polarization multiplexing. Light: Sci. Appl. 9:101. https://doi.org/10.1038/s41377-020-0327-7.

[35] Li Z, Chen C, Guan Z, Tao J, Chang S, Dai Q, Xiao Y, Cui Y, Wang Y, Yu S, Zheng G, Zhang S (2020) Three-Channel Metasurfaces for Simultaneous Meta-Holography and Meta-Nanoprinting: A Single-Cell Design Approach. Laser \& Photonics Reviews. 14:2000032. https://doi.org/10.1002/lpor.202000032.

[36] Han J, Intaravanne Y, Ma A, Wang R, Li S, Li Z, Chen S, Li J, Chen X (2020) Optical metasurfaces for generation and superposition of optical ring vortex beams. Laser \& Photonics Reviews 14:2000146. https://doi.org/10.1002/lpor.202000146.

[37] Intaravanne Y, Chen X (2020) Recent advances in optical metasurfaces for polarization detection and engineered polarization profiles. Nanophotonics 9:1003-1014. https://doi.org/10.1515/nanoph-2019-0479.

[38] Palik ED (1985) Handbook of Optical Constants of Solids. Academic, New York .

[39] Gan CH, Nash GR (2013) Broadband and efficient plasmonic control in the near-infrared and visible via strong interference of surface plasmon polaritons. Opt. Lett. 38:4453-4456. https://doi.org/10.1364/OL.38.004453.

[40] Bliokh KY, Rodríguez-Fortuño FJ, Nori F, Zayats AV (2015) Spin-orbit interactions of light. Nat. Photonics 9:796-808. https://doi.org/10.1038/nphoton.2015.201.

[41] Bao Y, Zu S, Liu W, Zhou L, Zhu X, Fang Z (2017) Revealing the spin optics in 
$\begin{array}{lllll}\text { conic-shaped metasurfaces. } & \text { Phys. Rev. B }\end{array}$ https://doi.org/10.1103/PhysRevB.95.081406.

[42] Zeng S, Zhang Q, Zhang X, Liu X, Xiao J (2018) Unidirectional excitation of plasmonic waves via a multilayered metal-dielectric-metal Huygens' nanoantenna. Opt. Lett. 43:3053-3056. https://doi.org/10.1364/OL.43.003053.

[43] Hwang Y, Yang JK (2019) Directional coupling of surface plasmon polaritons at $\begin{array}{lllll}\text { complementary } & \text { split-ring } & \text { resonators. } & \text { Sci. } & \text { Rep. }\end{array}$ https://doi.org/10.1038/s41598-019-43914-z.

[44] Yang Z, Fu Y, Yang J, Hu C, Zhang J (2018) Spin-encoded subwavelength all-optical logic gates based on single-element optical slot nanoantennas. Nanoscale 10:4523. https://doi.org/10.1039/C7NR08871J.

[45] Ying Z, Feng C, Zhao Z, Dhar S, Dalir H, Gu J, Cheng Y, Soref R, Pan DZ, Chen RT (2020) Electronic-photonic arithmetic logic unit for high-speed computing. Nat Commun. 11:2154. https://doi.org/10.1038/s41467-020-16057-3. 